\title{
Edge states propagating from a defect of graphite: Scanning tunneling spectroscopy measurements
}

\author{
Takahiro Kondo, Yujiro Honma, Junepyo Oh, Takahiro Machida, and Junji Nakamura* \\ Graduate School of Pure and Applied Sciences, University of Tsukuba, 1-1-1 Tennodai, Tsukuba, Ibaraki 305-8573 Japan
}

(Received 28 July 2010; revised manuscript received 16 September 2010; published 29 October 2010)

\begin{abstract}
We report nonuniform and long-range electronic perturbation of graphite near the Fermi level by the point defect based on the measurements with scanning tunneling spectroscopy. The states propagate with threefold symmetry perpendicular to the zigzag edges at the point defect, indicating the formation of the nonbonding states. The propagation continues for 3-4 $\mathrm{nm}$ from the defect and is accompanied by oscillations in the state energy and state intensity with a periodicity of $(\sqrt{3} \times \sqrt{3}) R 30^{\circ}$. The oscillation in the state intensity is ascribed to the disruption in the $\pi$-conjugated system in graphite around the defect, i.e., edge-states propagation from the defect while the oscillation in the state energy is ascribed to electron-electron interactions between the nonbonding $\pi$ electronic states and the standing-wave caused by $\pi$ bands of graphite.
\end{abstract}

DOI: $10.1103 /$ PhysRevB.82.153414

PACS number(s): 73.22.Pr, 07.79.Fc

The nonbonding $\pi$ electronic states of the graphite-related materials such as graphene sheets and graphite are of special interests to the wide variety of fields in science because they are the key electronic states in electronic devices ${ }^{1}$ and superconductor ${ }^{2}$ made of such materials and may be the active sites for a number of important chemical reactions ${ }^{3}$ such as oxygen-reduction reaction in fuel cells. ${ }^{4}$ These states are spontaneously formed near the zigzag edge of the graphite as an "edge state," which was first predicted by theory, ${ }^{5-7}$ and then observed experimentally by scanning tunneling microscopy (STM) ${ }^{8,9}$ It has also been reported that the similar local density of states of graphene or graphite near the Fermi level can appear due to different origins, such as the interference of quasiparticles, ${ }^{10}$ a Van Hove singularity, ${ }^{11}$ Landau quantization in the magnetic field, ${ }^{12}$ localization of the electronic states due to the stacking of two graphene layers, ${ }^{13}$ and $\mathrm{Pt}-\mathrm{C}$ hybridization. ${ }^{14}$ Understanding the origin and the spatial distribution of the new states near the Fermi level is vital to the future improvement of graphite-related technologies. In this Brief Report, the anisotropic propagation of the nonbonding $\pi$ electronic states has been observed around a defect at the graphite surface by scanning tunneling spectroscopy (STS) with a spatial resolution at the atomic scale.

All experiments were performed in an ultrahigh-vacuum STM chamber (UNISOKU, USM-1500) with a base pressure of $2 \times 10^{-10}$ Torr. Highly oriented pyrolytic graphite (HOPG) (ZYA grade) was cleaved in air using adhesive tape. Defects of graphite were created by $\mathrm{Ar}^{+}$-ion bombardment of HOPG at room temperature by using the commercial ion gun (ANELVA, 5-KV ion gun). The sample was bombarded with $\mathrm{Ar}^{+}$ions of $200 \mathrm{eV}$ at the normal incidence. The total ion doses on the sample were set to be lower than 1.0 $\times 10^{13}$ ions $/ \mathrm{cm}^{2}$, which has been measured independently by the Faraday cup. After the bombardment, the sample was heated to $900 \pm 100 \mathrm{~K}$ to remove any implanted Ar. The sample temperature during heating was measured by infrared thermometer. The sample was then cooled to $\approx 5 \mathrm{~K}$ with liquid helium. The surface morphology and the surface electronic structure were then examined by STM and STS, respectively, with a PtIr tip $(\mathrm{Pt}: \mathrm{Ir}=8: 2)$. STM images were recorded in the constant-current mode.

The atomic-scale STM image of the HOPG surface with a defect is shown in Fig. 1(a). It is well known that the $\beta$-carbon atoms of graphite appear as brighter spots in the
STM image. ${ }^{15}$ Here, the defect is shown at the center of the image. Around the defect, there are two characteristic features: (i) anisotropic propagation of a bright asteroidlike pattern with threefold symmetry and (ii) the $(\sqrt{3} \times \sqrt{3}) R 30^{\circ}$ superstructure appears around the asteroid region. This superstructure is confirmed by the Fourier transform image at the right top of Fig. 1(a). The asteroid pattern and superstructure have been reported previously as an image around a point vacancy defect at an $\alpha$ carbon on a graphite surface. ${ }^{16}$ We have measured STS spectra at many different points in the vicinity of the point defect. The right-hand side of Fig. 1(a) shows the spectra taken at the corresponding points A-D in Fig. 1(a). It is clear that the STS spectra changes with the position of the measurement. Spectrum A shows a parabolic shape corresponding to tails from the $\pi$ and $\pi^{*}$ bands of graphite, suggesting that the defect does not significantly perturb the electronic structure at positions like point $\mathrm{A}$. On the other hand, a huge $d I / d V$ peak appears at $\approx 160 \mathrm{mV}$ in the carbon spectrum around the defect at point $\mathrm{B}$, indicating that a new electronic state has appeared in the undisturbed surface due to the perturbation of the electronic states of graphite by the defect. Although the distance from the point defect to point $\mathrm{C}$ is the same as to point $\mathrm{B}$, there is no distinct $d I / d V$ peak in the spectrum $\mathrm{C}$. This suggests that the electronic perturbation propagates nonuniformly around the defect. In the direction toward B, i.e., the direction along the bright regions of the asteroid shape, the states are present even at the points far from the point defect, such as at point D.

To further investigate the propagation of the new states and the relationship to the defect structure, spatially resolved STS spectra (STS maps) were taken at several points. The peak intensity of the new states observed around the defect is summarized in Fig. 1(b). The examples of STS spectra are shown in Figs. 1(d)-1(i). New local density of states induced by the defect were found to propagate up to 3-4 $\mathrm{nm}$ away from the defect in three directions perpendicular to the short zigzag edges of the graphite surface at the defect. This is shown schematically with arrows in Fig. 1(c). The length of each zigzag edge at the defect is about $0.5 \mathrm{~nm}$. Zigzag edges are known to produce edge states at the second neighboring carbon atoms in graphene sheets ${ }^{5,7}$ or graphite. ${ }^{6}$ Such edge states have been predicted to be localized at the topmost layer of the graphite surface. ${ }^{6}$ The edge states are known as 

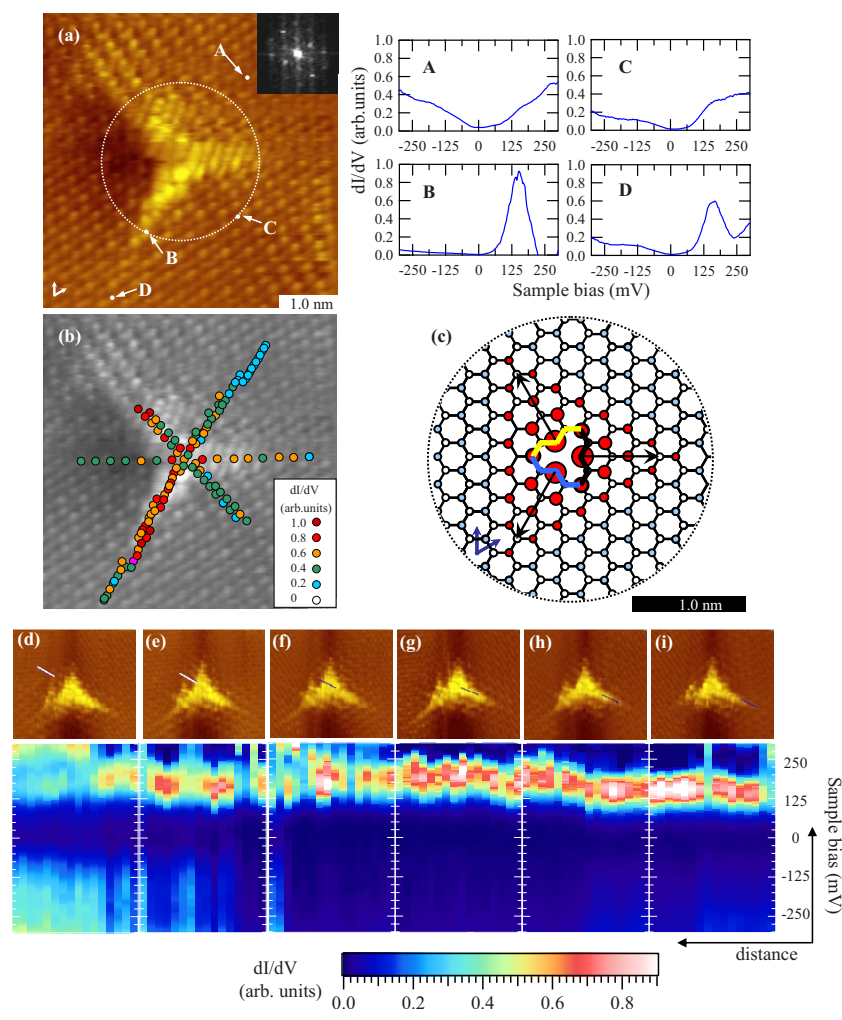

FIG. 1. (Color online) (a) STM image of the graphite surface with a point vacancy defect at $5.4 \mathrm{~K}\left(5.0 \times 5.0 \mathrm{~nm}^{2}\right.$, sample bias $V_{s}=301 \mathrm{mV}$, and tunneling current $\left.I_{t}=153 \mathrm{pA}\right)$. The inset shows two-dimensional Fourier transform of the image, where the spots forming the inner hexagon represent the $(\sqrt{3} \times \sqrt{3}) R 30^{\circ}$ superstructure observed in the vicinity of the defect. The STS spectra taken at the points A-D are represented at the right-hand side of the image. (b) Spatial distribution of the STS peak intensity observed around the defect, where the $d I / d V$ peak intensity in STS spectrum is plotted by color solid circle. (c) Schematic image of the point vacancy defect on graphite. Three zigzag edges at the defect are represented by solid zigzag lines. The size of the solid circle represents the intensity of the local density of states (edge states). The arrows perpendicular to the zigzag edge represent the propagation direction of the edge states. [(d)-(i)] Space-resolved STS spectra (STS map) taken at the line in the corresponding STM image. In each case, STS results from left to right at line are shown as STS map from left to right.

"nonbonding" states, a term which shall be used in this Brief Report hereafter. It is thus considered that the nonbonding states propagate out from the defect with the threefold symmetry indicated by the arrows in Fig. 1(c). Similar propagation of the modified electronic structure through the graphene sheet by defects has also been predicted by several groups based on theoretical calculations. ${ }^{17}$ The appearance of nonbonding peaks in the unoccupied region can be explained as follows: a point defect introduces dangling bonds at the three carbon atoms neighboring the vacancy. Each carbon atom should result in two dangling bonds, i.e., a $s p_{2}$ orbital ( $\sigma$ band) and a conjugated $p_{\mathrm{z}}$ orbital ( $\pi$ band). Since the $\sigma$ band is located at deeper energy levels than the $\pi$ band in graphite, two surplus electrons, freed by the point defect, should fill the $\sigma$-dangling bond. As a result of this electron
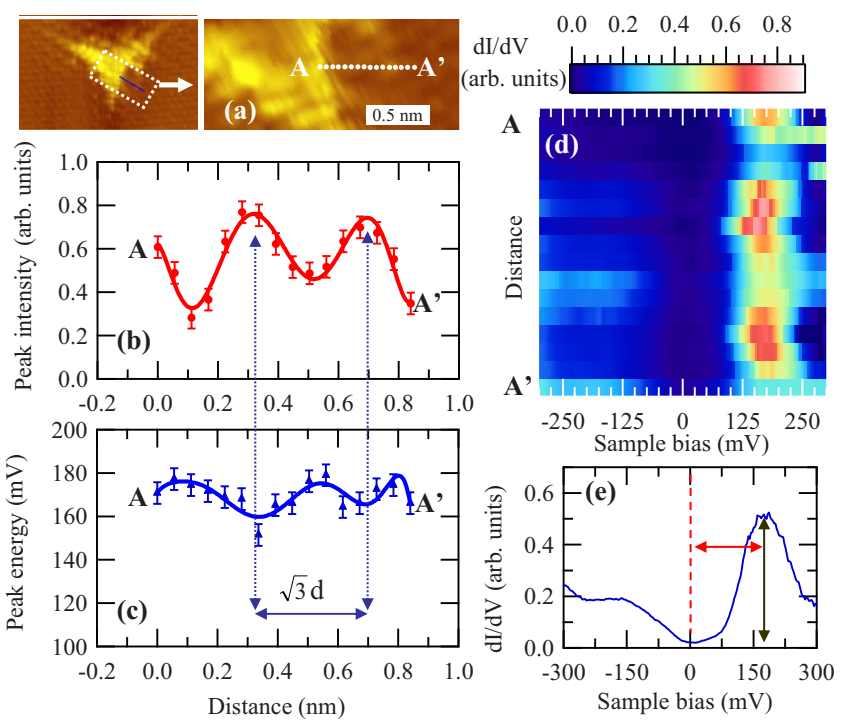

FIG. 2. (Color online) (a) STM image obtained during the STS measurements at $5.4 \mathrm{~K}\left(0.9 \times 2.8 \mathrm{~nm}^{2}, V_{s}=300 \mathrm{mV}\right.$, and $I_{t}$ $=150 \mathrm{pA})$. [(b) and (c)] Peak intensity and energy of the $d I / d V$ peak in the STS spectrum along $\mathrm{AA}^{\prime}$ in STM image $a$, respectively. The solid curve is a guide. " $d$ " represents the distance to the nearest-neighboring $\beta$-carbon atoms, $0.25 \mathrm{~nm}$. (d) Space-resolved STS spectra along A-A' in $a$. (e) The definitions of the peak intensity and energy of the STS spectrum are represented by red (dark gray) and black arrows, respectively.
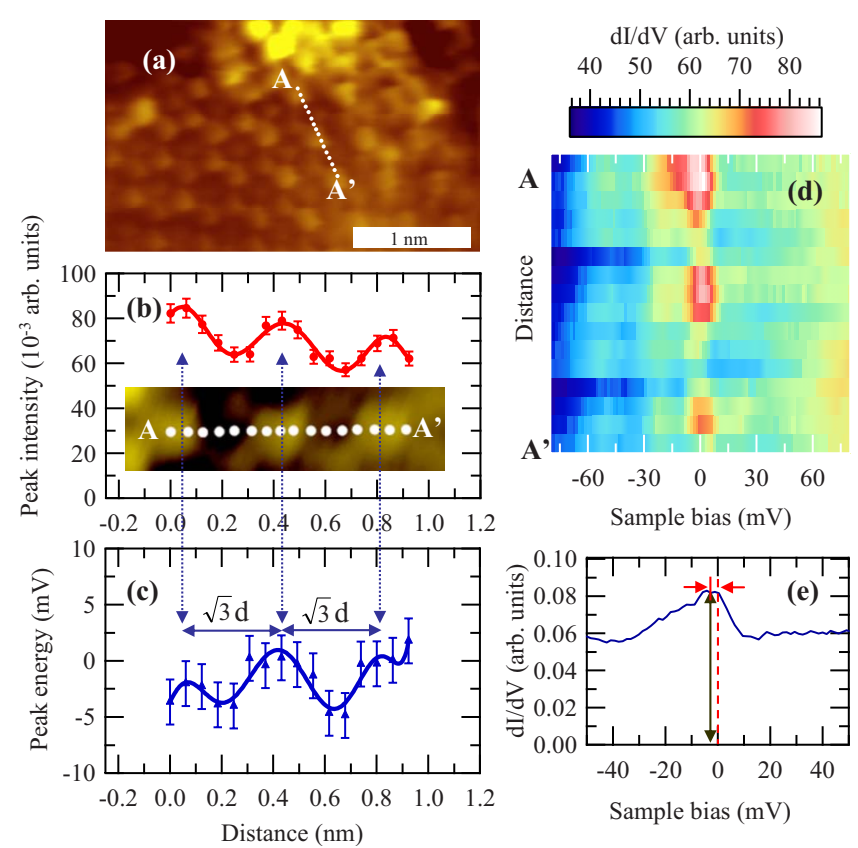

FIG. 3. (Color online) (a) STM image obtained during STS measurements at $5.3 \mathrm{~K}\left(2.0 \times 3.5 \mathrm{~nm}^{2}, V_{s}=150 \mathrm{mV}\right.$, and $I_{t}$ $=150 \mathrm{pA})$. [(b) and (c) [ Peak intensity and energy of the $d I / d V$ peak in the STS spectrum along $\mathrm{AA}^{\prime}$ in $a$, respectively. The solid curve is a guide. " $d$ " represents the distance to the nearestneighboring $\beta$-carbon atoms, $0.25 \mathrm{~nm}$. (d) Space-resolved STS spectra along $\mathrm{A}-\mathrm{A}^{\prime}$ in $a$. (e) The definitions of the peak intensity and energy of the STS spectrum are represented by red (dark gray) and black arrows, respectively. 


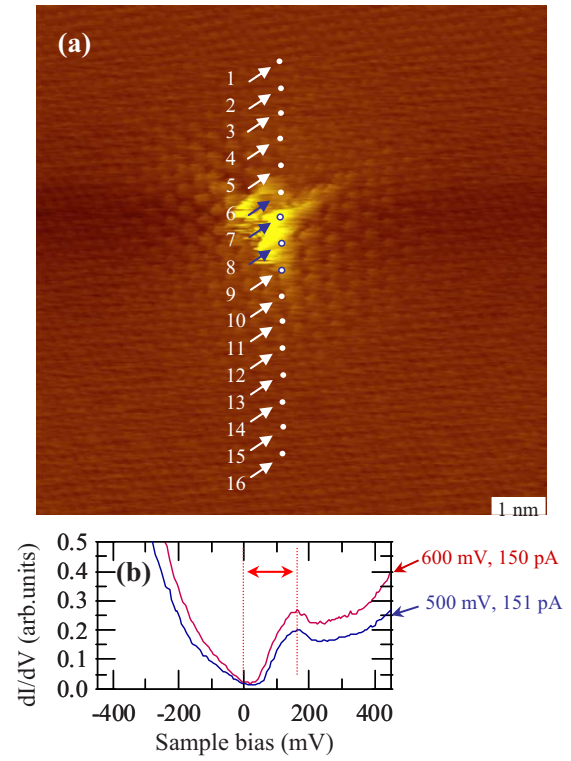

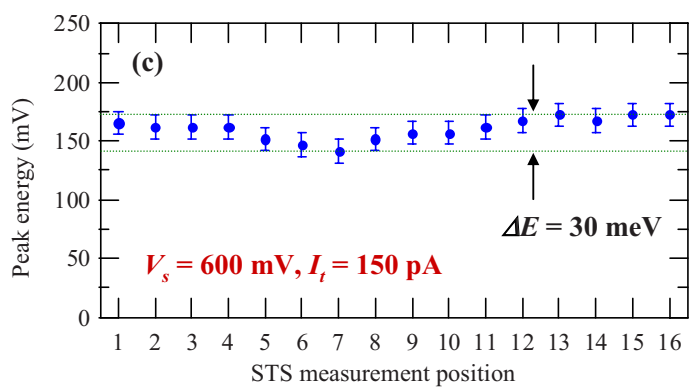

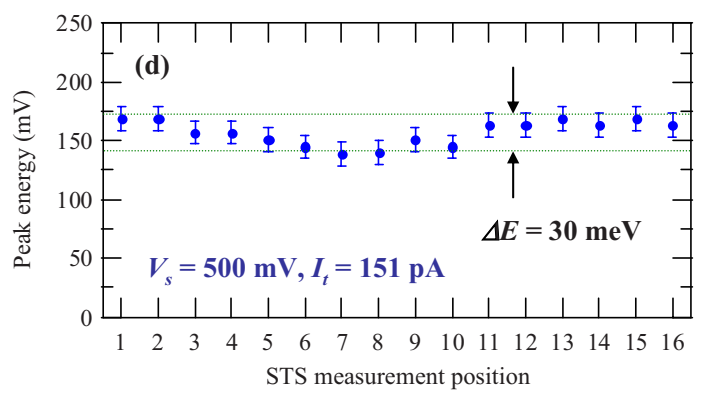

FIG. 4. (Color online) (a) STM image obtained during STS measurements at $5.3 \mathrm{~K}(10.0$ $\times 10.0 \mathrm{~nm}^{2}, V_{s}=600 \mathrm{mV}$, and $I_{t}$ $=150 \mathrm{pA}$ ). (b) STS results measured at the position 11 in $a$ with different tip-sample distances; (the results at the set points determined by $V_{s}=600 \mathrm{mV}$ and $V_{s}=500 \mathrm{mV}$ are shown). (c) Peak energy of the $d I / d V$ peak in the STS spectrum (arrow in $b$ ) at positions from 1 to 16 in $a$ at the tip-sample distance determined by the conditions of $V_{s}=600 \mathrm{mV}$ and $I_{t}=150 \mathrm{pA}$. (d) The same with $c$ except for the measurement condition of the tipsample distance $\left(V_{s}=500 \mathrm{mV}\right.$ and $\left.I_{t}=150 \mathrm{pA}\right)$. transfer, the nonbonding $\pi$-electronic state becomes unoccupied. A similar energy shift has also been reported based on the theoretical calculation. ${ }^{7}$

The other interesting features revealed in Figs. 1(d)-1(i) are the "oscillations" of the $d I / d V$ peak position and the peak intensity with the distance from the defect. The oscillations are shown in Fig. 2, which shows an identical defect to that in Fig. 1. The peak intensity [Fig. 2(b)] and the peak energy [Fig. 2(c)] oscillate with distance along $\mathrm{AA}^{\prime}$ in the STM image [Fig. 2(a)], where the intensity and the energy are defined by the arrows shown in Fig. 2(e). Along the line from $\mathrm{A}$ to $\mathrm{A}^{\prime}$ near the defect, both the peak intensity and the peak energy oscillate in an antiphase mode. With increasing the distance from the defect, the peak intensity becomes small. It should be noted that the periodicity of the oscillation almost corresponds to that of the $(\sqrt{3} \times \sqrt{3}) R 30^{\circ}$ superstructure, which is identical to the superstructure seen in the STM image. Furthermore, the atomic positions of the maxima in the intensity and minima of the energy correspond to the bright spots in the STM image as is also shown in Fig. 2. This accordance is observed in the STS measurements not only around point defects but also around defects of any size. The oscillations with the same periodicity in the intensity and the energy of the STS peaks are, however, not always antiphase. They are in phase if the STS peak appears in the occupied region. An example is shown in Fig. 3. Here, the defect in Fig. 3 is different from that in Fig. 1, in that the size of the defect in Fig. 3 is much larger. As shown in Fig. $3(\mathrm{e})$, the STS peak appears in the occupied region. ${ }^{18}$ In this particular case, the intensity and energy positions of STS peak oscillate with an in-phase mode [Figs. 3(b) and 3(c)], which is in contrast with those shown in Figs. 2(b) and 2(c).

Here, we discuss the origin of the oscillations. As shown in Figs. 2 and 3, the positions of the intensity peak maxima in the STS spectra correspond to those of brighter spots (modulated $\pi$-band states) in the STM image. Both have an identical $\sqrt{3}$ periodicity. That is, the spatial positions of the intense peaks of the nonbonding $\pi$ electronic states (edge states) are superimposed on the positions of the dense $\pi$-band states. It has been assumed that the origin of the $\sqrt{3}$ superstructure, as observed by STM, is due to the chargedensity oscillations of $\pi$ electrons similar to Friedel oscillations. ${ }^{16,19}$ In other words, the electron-scattering channel is opened between the $\mathrm{K}$ and $\mathrm{K}^{\prime}$ points in momentum space. The momentum change from $\mathrm{K}$ to $\mathrm{K}^{\prime}$ corresponds to $q=2 \mathrm{k}_{\mathrm{F}}$, leading to the $(\sqrt{3} \times \sqrt{3}) R 30^{\circ}$ structure in the local density of $\pi$ states. ${ }^{19}$ The nonbonding states are considered to be interacting with the $\pi$-band states forming a standing wave via an electron-electron interaction, which causes a shift in the energy level of the STS nonbonding peak toward the Fermi level, i.e., larger peak intensities lead to larger electron-electron interactions. With increasing distance from the defect, the oscillation amplitude of the peak intensity becomes small in both types of defect [Figs. 1(b)-1(i) and $3(\mathrm{~b})]$. The propagating states finally disappear about 3-4 nm from the defect. Theoretical calculations are required to prove the proposed model of the oscillations. There are similar reports of STS peak oscillation due to the potential modulation via Friedel oscillation and/or the standing wave on $\mathrm{Si}(111) \sqrt{3} \times \sqrt{3} \mathrm{Ag} .{ }^{20}$ These are not the case as the origin of the energy and intensity oscillations in Figs. 2 and 3 because negligible peak intensity is detected even at the position above the $(\sqrt{3} \times \sqrt{3}) R 30^{\circ}$ superstructure [see, e.g., Fig. 1(c)] which is due to the nonuniform propagation of the states. That is, the propagation of the nonbonding states and that of the standing wave are different. One may also suspect that the origin of the oscillation should be originated from the difference in the tip-sample distance because of the difference in the $\pi$-band charge density. Indeed, the STM tip is transferred to different positions during STS-map measurement in the constant-current mode. It is thus possible that the tip-sample distance is different due to the difference in the charge density. However, the intensity oscillation in Fig. 3 is not the case because the STS peak intensity is high at the bright position in the STM image. That is, the STS peak is intense when the tip-sample distance is large. It is thus considered that the bright spots in the STM images are ascribed to the distinct state near $E_{f}$ because the STM images mea- 
sured at the bias voltage of $150 \mathrm{meV}$ reflect the new state intensity significantly in addition to the $\pi^{*}$ states from $E_{f}$ to $150 \mathrm{meV}$. As for the energy oscillation, it was confirmed that peak energy did not change at different tip-sample distance as shown in Fig. 4, where the STS results measured at the different tip-sample distances were summarized. STS peak energy depends on the position on the sample but is almost independent of the tip-sample distance. ${ }^{21}$

In summary, STS has been used to show disruptions in the $\pi$-conjugated system near the Fermi level propagate away from a point defect in a graphite surface with the same periodicity of the $(\sqrt{3} \times \sqrt{3}) R 30^{\circ}$ structure. The distinct local density of the nonbonding states propagates in three directions up to 3-4 $\mathrm{nm}$ from the defect. The direction of the state's propagation is perpendicular to the three zigzag edges formed at the vacancy. Each short zigzag edge thus generates the nonbonding states. The STS peak positions of the non- bonding states oscillate through space with a periodicity of $\sqrt{3}$. As well as the peak intensity, the peak energy of the STS peak oscillates as a function of the distance from the defect of graphite, which is explained by electron-electron interactions between the nonbonding $\pi$ electronic states and the standing wave caused by $\pi$ bands of graphite. The observed large and nonuniform perturbation of the electronic structure of the graphite surface around the defect indicates a significant change in the physical and chemical properties of the graphite surface. This strongly suggests the possibility of new carbon-based materials based on the control of such electronic perturbations by defects and/or dopants.

Note added. At this point we would like to note that we recently became aware of a related work on the STS observation around the point defect of graphite by Ugada et al. ${ }^{22}$

We appreciate financial supports from NEDO (New Energy and Industrial Technology Development).
*Corresponding author; nakamura@ims.tsukuba.ac.jp

${ }^{1}$ A. H. Castro Neto et al., Rev. Mod. Phys. 81, 109 (2009); K. A. Ritter and J. W. Lyding, Nature Mater. 8, 235 (2009); D. A. Areshkin et al., Nano Lett. 7, 204 (2007); E. J. H. Lee et al., ibid. 3, 486 (2008); Y. Yoon and J. Guo, Appl. Phys. Lett. 91, 073103 (2007); D. Basu et al., ibid. 92, 042114 (2008); S. Banerjee, M. Sardar, N. Gayathri, A. K. Tyagi, and B. Raj, Phys. Rev. B 72, 075418 (2005).

${ }^{2}$ K. Sugawara, Nat. Phys. 5, 40 (2009); M. S. Dresselhaus and G. Dresselhaus, Adv. Phys. 51, 1 (2002).

${ }^{3}$ K. Takahara et al., Phys. Rev. B 76, 035442 (2007); D. Jiang et al., J. Chem. Phys. 126, 134701 (2007).

${ }^{4}$ S. F. Huang et al., Phys. Rev. B 80, 235410 (2009).

${ }^{5}$ S. E. Stein and R. L. Brown, J. Am. Chem. Soc. 109, 3721 (1987); K. Kobayashi, Phys. Rev. B 48, 1757 (1993); D. J. Klein, Chem. Phys. Lett. 217, 261 (1994); M. Fujita et al., J. Phys. Soc. Jpn. 65, 1920 (1996); K. Nakada, M. Fujita, G. Dresselhaus, and M. S. Dresselhaus, Phys. Rev. B 54, 17954 (1996).

${ }^{6}$ Y. Miyamoto, K. Nakada, and M. Fujita, Phys. Rev. B 59, 9858 (1999).

${ }^{7}$ T. Kawai, Y. Miyamoto, O. Sugino, and Y. Koga, Phys. Rev. B 62, R16349 (2000).

${ }^{8}$ Z. Klusek et al., Appl. Surf. Sci. 161, 508 (2000); P. L. Giunta and S. P. Kettl, J. Chem. Phys. 114, 1807 (2001).

${ }^{9}$ Y. Niimi et al., Appl. Surf. Sci. 241, 43 (2005); Y. Kobayashi, K.-i. Fukui, T. Enoki, K. Kusakabe, and Y. Kaburagi, Phys. Rev. B 71, 193406 (2005); Y. Niimi, T. Matsui, H. Kambara, K. Tagami, M. Tsukada, and H. Fukuyama, ibid. 73, 085421 (2006).

${ }^{10}$ G. M. Rutter et al., Science 317, 219 (2007).

${ }^{11}$ G. Li et al., Nat. Phys. 6, 109 (2010).

${ }^{12}$ T. Matsui, H. Kambara, Y. Niimi, K. Tagami, M. Tsukada, and H. Fukuyama, Phys. Rev. Lett. 94, 226403 (2005).

${ }^{13}$ P. Lauffer et al., Phys. Rev. B 77, 155426 (2008).

${ }^{14}$ T. Kondo, Y. Iwasaki, Y. Honma, Y. Takagi, S. Okada, and J.
Nakamura, Phys. Rev. B 80, 233408 (2009).

${ }^{15}$ D. Tománek, S. G. Louie, H. J. Mamin, D. W. Abraham, R. E. Thomson, E. Ganz, and J. Clarke, Phys. Rev. B 35, 7790 (1987) $\beta$-carbon atoms are the carbon atoms located above the centers of the hexagons of the layer beneath; the remaining carbon atoms are $\alpha$-carbon atoms.

${ }^{16}$ K. F. Kelly et al., Science 273, 1371 (1996); K. F. Kelly and N. J. Halas, Surf. Sci. 416, L1085 (1998); J. G. Kushmerick et al., J. Phys. Chem. B 103, 1619 (1999).

${ }^{17}$ T. O. Wehling et al., Chem. Phys. Lett. 476, 125 (2009); X. Yang et al., Carbon 47, 1399 (2009); S. Casolo et al., J. Chem. Phys. 130, 054704 (2009); O. V. Yazyev and L. Helm, Phys. Rev. B 75, 125408 (2007); H. Amara, S. Latil, V. Meunier, Ph. Lambin, and J.-C. Charlier, ibid. 76, 115423 (2007); A. A. ElBarbary, R. H. Telling, C. P. Ewels, M. I. Heggie, and P. R. Briddon, ibid. 68, 144107 (2003).

${ }^{18}$ In our bombardment condition, larger size defects were also observed as well as the point vacancy defect on the surface. Around $65 \%$ of the observed larger size defects, STS peaks are detected at occupied region, $17 \%$ are unoccupied peak, $14 \%$ are both occupied and unoccupied peaks, and $4 \%$ are no peak. Peak energy is not always the same but appears in the range from 0 to $\pm 200 \mathrm{meV}$. For such larger size defects, the origin of the stateenergy position is not clear due to the complicated structure of such defect.

${ }^{19}$ H. A. Mizes and J. S. Foster, Science 244, 559 (1989); P. Ruffieux et al., Phys. Rev. B 71, 153403 (2005).

${ }^{20}$ M. Ono, Y. Nishigata, T. Nishio, T. Eguchi, and Y. Hasegawa, Phys. Rev. Lett. 96, 016801 (2006); Y. Hasegawa et al., J. Phys.: Conf. Ser. 61, 399 (2007); M. Ono et al., Appl. Surf. Sci. 256, 469 (2009).

${ }^{21}$ See supplementary material at http://link.aps.org/supplemental/ 10.1103/PhysRevB.82.153414 for the effect of the tip-sample distance on the STS results.

${ }^{22}$ M. M. Ugeda et al., Phys. Rev. Lett. 104, 096804 (2010). 